\title{
Granulocyte Colony-stimulating Factor Administration to HIV-infected Subjects Augments Reduced Leukotriene Synthesis and Anticryptococcal Activity in Neutrophils
}

\author{
Michael J. Coffey, ${ }^{\star}$ Susan M. Phare, ${ }^{*}$ Sarah George, ${ }^{\ddagger}$ Marc Peters-Golden, ${ }^{\star}$ and Powel H. Kazanjian ${ }^{\ddagger}$ \\ Divisions of *Pulmonary and Critical Care Medicine and ${ }^{\ddagger}$ Infectious Disease, University of Michigan Medical Center, Ann Arbor, \\ Michigan 48109
}

\begin{abstract}
Neutrophil (PMN) dysfunction occurs in HIV infection. Leukotrienes (LT) are mediators derived from the 5-lipoxygenase (5-LO) pathway that play a role in host defense and are synthesized by PMN. We investigated the synthesis of LT by PMN from HIV-infected subjects. There was a reduction (4.0 $\pm 1.3 \%$ of control) in LT synthesis in PMN from $\mathrm{HIV}$-infected compared with normal subjects. This was associated with reduced expression of 5-LO-activating protein (31.2 $\pm 9.6 \%$ of normal), but not of 5-LO itself. Since HIV does not directly infect PMN, we considered that these effects were due to reduced release of cytokines, such as granulocyte colony-stimulating factor (G-CSF). We examined the effect of G-CSF treatment (300 $\mu \mathrm{g}$ daily for $5 \mathrm{~d}$ ) on eight $\mathrm{HIV}$-infected subjects. PMN were studied in vitro before therapy (day 1) and on days 4 and 7 . $\mathrm{LTB}_{4}$ synthesis was increased on day 4 of G-CSF treatment, and returned toward day 1 levels on day 7. 5-LO and 5-LO-activating protein expression were increased in parallel. As a functional correlate to this increase in PMN LT synthesis by G-CSF, we examined the effects on killing of Cryptococcus neoformans. Anticryptococcal activity of PMN from HIV-infected subjects was less than that of PMN from normal subjects. G-CSF treatment improved fungistatic activity of PMN. This increase in antifungal activity was attenuated by in vitro treatment with the LT synthesis inhibitor, MK-886. In conclusion, PMN from HIV-infected subjects demonstrate reduced 5-LO metabolism and antifungal activity in vitro, which was reversed by in vivo G-CSF therapy. (J. Clin. Invest. 1998. 102:663-670.) Key words: AIDS • eicosanoids • prostaglandins $\bullet$ cytokines $\bullet$ opportunistic infections
\end{abstract}

\section{Introduction}

HIV infection results in increased susceptibility to opportunistic and nonopportunistic infections. Although defects in cell-

Address correspondence to Michael J. Coffey, M.D., Assistant Professor of Internal Medicine, University of Michigan Medical Center, 6301 MSRB III, 1150 W. Medical Center Dr., Ann Arbor, MI 481090642. Phone: 734-764-4554; FAX: 734-764-4556; E-mail: mcoffey@ umich.edu

Received for publication 25 October 1997 and accepted in revised form 19 June 1998.

J. Clin. Invest.

(C) The American Society for Clinical Investigation, Inc. 0021-9738/98/08/0663/08 \$2.00

Volume 102, Number 4, August 1998, 663-670

http://www.jci.org mediated immunity are prominent (1), there is also evidence of compromised neutrophil (PMN) function (2). For example, PMN from HIV-infected subjects with AIDS demonstrate reduced chemotaxis compared with control PMN (2). Furthermore, phagocytosis and killing of Staphylococcus aureus were significantly reduced compared with controls (3). Finally, PMN superoxide activity, which is instrumental in microbicidal activity, was also shown to be depressed in PMN from HIVinfected subjects $(4,5)$. Clinically, impaired PMN function in these patients is associated with an increased prevalence of pyogenic infections (6).

Leukotrienes $(\mathrm{LT})^{1}$ are products of the 5-lipoxygenase (5-LO) pathway of arachidonic acid (AA) metabolism $(7,8)$. They are generated in large amounts by $\mathrm{PMN}$, and play an important role in host-defense mechanisms $(9,10)$. LT enhance phagocytosis (11), expression of cell surface CR3 molecules (12), and the secretion of $\mathrm{O}_{2}^{-}$and lysosomal hydrolases (10). Mice whose 5-LO gene has been disrupted, and are therefore LT-deficient, exhibited impaired pulmonary bacterial clearance and increased mortality after intratracheal challenge with Klebsiella pneumoniae (13). Alveolar macrophages (AM) from these 5-LO knockout animals also demonstrated a reduced capacity to phagocytose and kill bacteria in vitro. Our laboratory has recently demonstrated that 5-LO metabolism is reduced in AM from HIV-infected subjects (14). We speculated that a similar defect in LT synthesis in PMN might reduce PMN antimicrobial activity, thereby leading to further compromise of the immune system of these HIV-infected patients.

Granulocyte colony-stimulating factor (G-CSF) has been used in HIV disease to treat neutropenia induced by antiretroviral agents, antibiotics, and overwhelming infection (15). It has been shown to upregulate PMN killing ability against various microorganisms in vitro (3). In vitro incubation with the related cytokine granulocyte-macrophage CSF (GM-CSF) has been shown to augment 5-LO metabolism in normal PMN by increasing expression of 5-LO and its helper protein, 5-LO-activating protein, termed "FLAP" $(16,17)$. G-CSF treatment in vitro has been shown to increase AA release in PMN (18). However, the effect of G-CSF on PMN 5-LO metabolism has not been investigated, either in vitro or in vivo. In this study, we determined 5-LO metabolism in PMN from HIV-infected subjects isolated before and after in vivo treatment with G-CSF. We also examined the effect of G-CSF administration on PMN antifungal activity.

\footnotetext{
1. Abbreviations used in this paper: AA, arachidonic acid; CFU, colony-forming unit; COX, cyclooxygenase; 5-LO, 5-lipoxygenase; FLAP, 5-LO-activating protein; G-CSF, granulocyte colony-stimulating factor; GM-CSF, granulocyte-macrophage colony-stimulating factor; LT, leukotriene; PG, prostaglandin; $\mathrm{PLA}_{2}$, phospholipase $\mathrm{A}_{2}$; TBS, tris-buffered saline.
} 


\section{Methods}

Clinical study protocol. We carried out a prospective trial of G-CSF therapy in eight HIV-infected subjects with low $\left(<100 \mathrm{~cm}^{2}\right) \mathrm{CD} 4$ counts without evidence of opportunistic infection. The experimental protocol was approved by the University of Michigan Institutional Review Board for Approval of Research Involving Human Subjects. Full informed consent was obtained from all subjects before the study. All subjects were on antiretroviral, but not on antifungal, therapy. Subjects were treated with G-CSF at a dose of $300 \mu \mathrm{g}$ subcutaneously on each of days $1-5$. PMN were isolated on day 1 , before G-CSF therapy, and day 7, $2 \mathrm{~d}$ after stopping G-CSF. Four subjects were also studied on day 4 . Each subject acted as his or her own control. Total white cell and absolute PMN counts were performed on days 1, 4, and 7. In addition, HIV load, as measured by (branched) bDNA (19), was determined before and after the drug study. Subjects were carefully monitored for adverse events during the study. PMN 5-LO metabolism and anticryptococcal activity in vitro were also determined. HIV-negative control subjects without evidence of systemic infection were also studied. These subjects who were recruited from the employees at University Hospital (Ann Arbor, MI), were normal volunteers, both male and female, who did not smoke and were taking no medication.

Isolation of PMN. PMN were isolated from venous blood drawn from HIV-infected subjects with low CD4 counts and from normal volunteers. A volume of $5 \mathrm{ml}$ of heparinized whole blood was layered over $3.5 \mathrm{ml}$ of a mixture of sodium metrizoate and Ficoll (1-Step Polymorphs; Accurate Chemical \& Scientific Corporation, Westbury, $\mathrm{NY}$ ) in a $15-\mathrm{ml}$ centrifuge tube. The sample was centrifuged at $450 \mathrm{~g}$ for $30 \mathrm{~min}$ in a swing-out rotor at $22^{\circ} \mathrm{C}$. After centrifugation, the distinct lower PMN-containing band was aspirated and diluted in $0.45 \%$ $\mathrm{NaCl}$ solution to restore normal osmolality. The cells were washed twice in $0.5 \mathrm{~N}$ saline and resuspended in Iscove's medium. This resulted in $<5 \%$ contamination with erythrocytes and $>95 \% \mathrm{PMN}$ as determined by Diff-Quik staining.

Quantitation of maximal PMN LT synthetic capacity. Freshly isolated PMN were suspended in Iscove's medium at $1 \times 10^{6} / \mathrm{ml}$ in Eppendorf tubes and incubated for 30 min with $10 \mu \mathrm{M}$ calcium ionophore (A23187; Calbiochem Corp., La Jolla, CA) or vehicle $(0.5 \%$ DMSO) alone. The cells were then centrifuged and the supernatant was frozen at $-70^{\circ} \mathrm{C}$ for subsequent $\mathrm{LTB}_{4}$ and prostaglandin $(\mathrm{PG}) \mathrm{E}_{2}$ analysis by enzyme immunoassay (Cayman Chemical, Ann Arbor, MI). To directly compare EIA results from controls and HIV-infected subjects, the sample from each HIV-infected subject was analyzed in parallel with that of a control subject studied on the same day. For each sample, the average of duplicate determinations was calculated. Data are expressed as picograms product per $1 \times 10^{6}$ cells.

Analysis of $\left[{ }^{3} \mathrm{H}\right] A A$ release and metabolism. Isolated PMN from control and HIV-infected subjects were incubated overnight. As measured by trypan blue exclusion, viability of PMN was: normal subjects $87.7 \pm 1.5 \%$ at $24 \mathrm{~h}$, and $55.6 \pm 6.8 \%$ at $48 \mathrm{~h}$; HIV-infected subjects was $77.3 \pm 4.7 \%$ at $24 \mathrm{~h}$, and $47.6 \pm 0.1 \%$ at $48 \mathrm{~h}(P=\mathrm{NS}, n=3)$. Release and metabolism of AA was assessed in PMN whose lipids were prelabeled by incubation with $\left[{ }^{3} \mathrm{H}\right] \mathrm{AA}$. This was accomplished by including $0.5 \mu \mathrm{Ci}\left[{ }^{3} \mathrm{H}\right] \mathrm{AA}(60-100 \mathrm{Ci} / \mathrm{mmol} \mathrm{sp}$ act) (Dupont-New England Nuclear, Boston, MA) in the medium during culture for $1.5 \mathrm{~h}$. Cells were then centrifuged and washed, and AA metabolism was determined by incubation in Iscove's medium for 30 min with $10 \mu \mathrm{M}$ A23187. To assess total AA release, cells were stimulated with A23187 in the presence of $0.1 \%$ BSA and radioactivity in the medium was determined (20). Cell supernatants were extracted using $\mathrm{C}_{18} \mathrm{Sep}$ Paks, Waters Corp., Milford, MA, and radiolabeled eicosanoids were separated by reverse-phase HPLC (20), identified by co-elution with authentic standards (Cayman Chemicals, Ann Arbor, MI), and quantitated using an on-line radioactivity detector (Radiomatic model 515; Packard Instruments, Downers Grove, IL). Radiolabeled products released were expressed as a percentage of radioactivity incorporated into cellular membrane phospholipids.
Immunoblot analysis of total cellular 5-LO, FLAP, and phospholipase $A_{2}\left(P L A_{2}\right)$. Steady-state quantities of 5-LO, FLAP, and cytosolic $\mathrm{PLA}_{2}\left(\mathrm{cPLA}_{2}\right)$ proteins were assessed by immunoblot analysis using a modification of methods described previously (21). Briefly, equal amounts of protein $(5-20 \mu \mathrm{g})$ were separated on $10 \%$ SDSpolyacrylamide gels by the method of Laemmli (22). High and low molecular weight rainbow markers (Amersham Chemical Corp., Arlington Heights, IL) were also loaded on each gel. After overnight transfer to nitrocellulose membranes (Bio-Rad Laboratories, Richmond, CA), blots were blocked by incubating for $1 \mathrm{~h}$ with $10 \%$ nonfat dried milk in Tris-buffered saline (TBS), washed in TBS containing $0.1 \%$ Tween (TBS-T), and incubated at room temperature for $1 \mathrm{~h}$ with rabbit polyclonal antisera raised against either human leukocyte 5-LO (1:3,000 dilution), amino acid residues 41-52 of the human FLAP sequence (1:5,000 dilution), or recombinant human $\mathrm{CPLA}_{2}$ (1:1,000 dilution). Antisera against 5-LO and FLAP were provided by Dr. J. Evans, Merck Frosst (Pointe Claire-Dorval, Quebec); anticPLA $_{2}$ antiserum (23) was provided by Dr. J. Clark (Genetics Institute, Cambridge, MA). After washing, blots were incubated for $1 \mathrm{~h}$ with horseradish peroxidase-conjugated goat anti-rabbit IgG (Amersham Chemical Corp.) at a dilution of 1:5,000 in TBS-T. Membranes were then washed and developed using the enhanced chemiluminescent Western blotting system (Amersham Chemical Corp.). Multiple exposures of each blot were obtained. Densities of luminescent bands were quantitated in appropriately exposed autoradiographs by video densitometry using National Institutes of Health Image software. Samples obtained from controls and HIV-infected subjects were loaded on the same gel, and underwent immunoblot analysis in parallel.

Anticryptococcal activity of PMN. The strain of Cryptococcus neoformans (C. neoformans) H99/C3D (American Type Culture Connection, Rockville, MD) was used because it has been isolated from human cerebral spinal fluid and the size of its capsule does not increase in response to physiological concentrations of $\mathrm{CO}_{2}$ in tissue culture (24). C. neoformans was stored on Sabouraud's dextrose agar slants (Difco Laboratories, Detroit, MI) and passaged monthly to fresh slants. Before inoculation, C. neoformans was prepared by resuspending a slant in Sabouraud's broth medium containing $1 \%$ neopeptone and $2 \%$ dextrose and incubating at $35^{\circ} \mathrm{C}$ for $3 \mathrm{~d}$. It was washed three times in sterile water, counted, and resuspended in culture medium. $2 \times 10^{4}$ organisms were added to $2 \times 10^{5}$ cells per 200 $\mu \mathrm{l}$ well. After coincubation with PMN for $48 \mathrm{~h}$, the cryptococcal burden was assessed, as described (24). Briefly, cells were lysed with $0.1 \%$ Triton X-100 to release intracellular organisms. The number of colony-forming units (CFU) were determined by plating serial dilutions of the lysates in quadruplicate on Sabouraud's dextrose agar, which were cultured in $5 \% \mathrm{CO}_{2}$ at $35^{\circ} \mathrm{C}$.

C. neoformans grew well in media alone. 18,000 $\pm 1,414.4$ CFU plated at time 0 in media alone increased after $48 \mathrm{~h}$ to a mean CFU of 330,000 $\pm 51,600 \mathrm{CFU}$ (18.3-fold increase, $P<0.05, n=4)$ ). Although, we did not measure time 0 CFU in every experiment, our CFU at $48 \mathrm{~h}$ were always in the $3-5 \times 10^{5}$ range increasing from a baseline of $2 \times$ $10^{4}$ organisms added to culture media at time 0 , indicating significant growth of $C$. neoformans. Percent change of $C$. neoformans growth compared with control medium was calculated as ([\{CFU experimental group $\}-\{\mathrm{CFU}$ control $\}] /\{\mathrm{CFU}$ control $\}) \times 100$. When this formula is used, suppression of $C$. neoformans growth by neutrophils is reflected by a negative change in C. neoformans compared with medium alone. Positive values indicate increased growth of the fungus when cocultured with neutrophils compared with medium alone. This formula is similar to that used by Chen et al. (24), where they include data for time $0 \mathrm{CFU}$ as well as $48 \mathrm{~h}$ in each experiment. Using their formula values of $-100 \%$ change in $\mathrm{CFU}$ are equivalent to fungicidal activity, whereas values between 0 and $-99 \%$ are in the fungistasis range (24). When we utilized the formula of Chen et al. we only detected fungistasis, and not fungicidal activity, after incubation of $C$. neoformans with PMN. Using this in vitro assay of anticryptococcal activity, we were able to detect consistent antifungal activity incubating PMN from normal subjects with C. neoformans for $24 \mathrm{~h}$ 


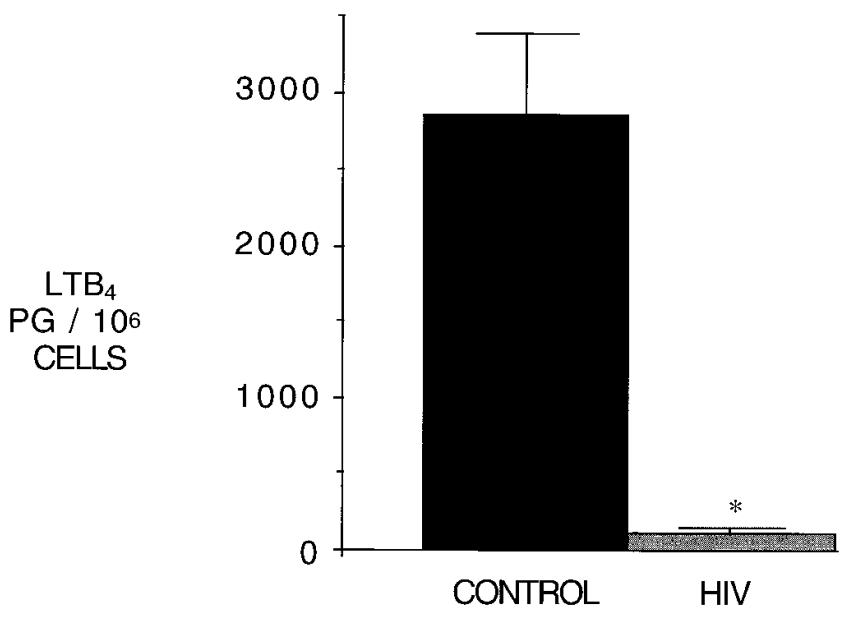

Figure 1. $\mathrm{LTB}_{4}$ synthesis in PMN from control and HIV-infected subjects. PMN were isolated as described in Methods and plated at $1 \times$ $10^{6} / \mathrm{ml}$ in Iscove's medium. Maximal $\mathrm{LTB}_{4}$ release was determined after stimulation with $\mathrm{A} 23187(10 \mu \mathrm{M})$ for $30 \mathrm{~min}$ at $37^{\circ} \mathrm{C}$. Medium was analyzed for $\mathrm{LTB}_{4}$ by EIA, and expressed as picograms per $10^{6}$ cells. $n=10, * P<0.001$

( $-37.5 \pm 16.5 \%$ of $C$. neoformans growth alone, $P=0.04$ compared with $5 \mathrm{~h}, n=4)$ and $48 \mathrm{~h}(-33.3 \pm 17.7 \%$, with no fungistasis demonstrated at $5 \mathrm{~h}(35.3 \pm 23.1 \%)$. Therefore, we used the $48 \mathrm{~h}$ time point in subsequent in vitro anticryptococcal assays. The FLAP inhibitor, MK-886 (a kind gift from P. Vickers [Merck Frosst, Quebec]) was added to cultures at a concentration of $1 \mu \mathrm{M}$ in selected experiments.

Data analysis. Where indicated, data were expressed as the mean \pm SEM. Differences between the mean values of HIV-infected and control groups were analyzed by ANOVA, with statistical significance assessed by the Scheffe test. A $P$ value $<0.05$ was considered significant.

\section{Results}

AA metabolism in PMN from HIV-infected subjects. Fig. 1 shows the maximal LT synthetic capacity from endogenous AA of PMN obtained from control and AIDS patients, assessed by quantitating immunoreactive $\mathrm{LTB}_{4}$ synthesis after A23187 activation. $\mathrm{LTB}_{4}$ synthesis was dramatically reduced in PMN from HIV-infected subjects to $4 \pm 1.3 \%$ of normal PMN levels. To confirm the EIA results demonstrating reduced 5-LO metabolic capacity in PMN from HIV-infected subjects, we performed HPLC analysis of the radiolabeled products released by prelabeled cells from control and HIV-infected volunteers stimulated with A23187. A representative eicosanoid profile of A23187-stimulated PMN from control subjects is shown in Fig. $2 A$. It consists predominantly of 5-LO products $\left(\mathrm{LTB}_{4}\right.$ and 5-hydroxyeicosatetraenoic acid [5-HETE]), with lesser amounts of the cyclooxygenase (COX) products, $\mathrm{PG}$. By contrast, PMN from HIV-infected subjects with low CD4 counts (Fig. $2 \mathrm{~B}$ ) synthesized substantially lesser amounts of $\left[{ }^{3} \mathrm{H}\right] 5-\mathrm{LO}$ products than did control PMN and 5-LO products represented only a minority of the $\left[{ }^{3} \mathrm{H}\right] \mathrm{AA}$ released. Importantly, the HPLC data demonstrate reduction not only in $\mathrm{LTB}_{4}$, but also 5-HETE, indicating a defect common to the 5-LO pathway as a whole.

One possible explanation for the reduction in 5-LO metabolic capacity of PMN from these HIV-infected subjects could be a decrease in the $\mathrm{PLA}_{2}$-mediated availability of AA, the substrate for LT synthesis. However, PMN from HIV-infected subjects released similar (Table I) total quantities of $\left[{ }^{3} \mathrm{H}\right] \mathrm{AA}$ in response to A23187 as did PMN from control subjects (percent of incorporated counts). This finding points to a postphospholipase step as the cause of the reduced 5-LO metabolic capacity of PMN from HIV-infected subjects.

5-LO and FLAP expression in PMN from HIV-infected subjects. Reduced 5-LO metabolic capacity in PMN from $\mathrm{HIV}$-infected subjects might reflect altered expression of 5-LO and/or FLAP proteins in these cells. To examine this possibility, Western blot analysis for 5-LO and FLAP was performed on crude lysates of PMN from control and HIV-infected subjects (Fig. 3). Cells from HIV-infected subjects exhibited a reduction in FLAP expression relative to control subjects (taken as $100 \%$ ). By contrast there was no change in 5-LO expression compared with control subjects. The expression of cPLA $\mathrm{PL}_{2}$ protein (not shown) was unchanged in PMN from HIV-infected subjects $(76.25 \pm 19.7 \%$ of control, $n=8)$ as compared with those obtained from control subjects, in keeping with the similar magnitude of AA release in the two groups.

Anticryptococcal activity of PMN from HIV-infected subjects. To examine the functional significance of this defect in 5-LO metabolism, we investigated the ability of PMN from the two groups to kill C. neoformans, a frequent opportunistic infection in the lung and the most common cause of meningitis
NORMAL

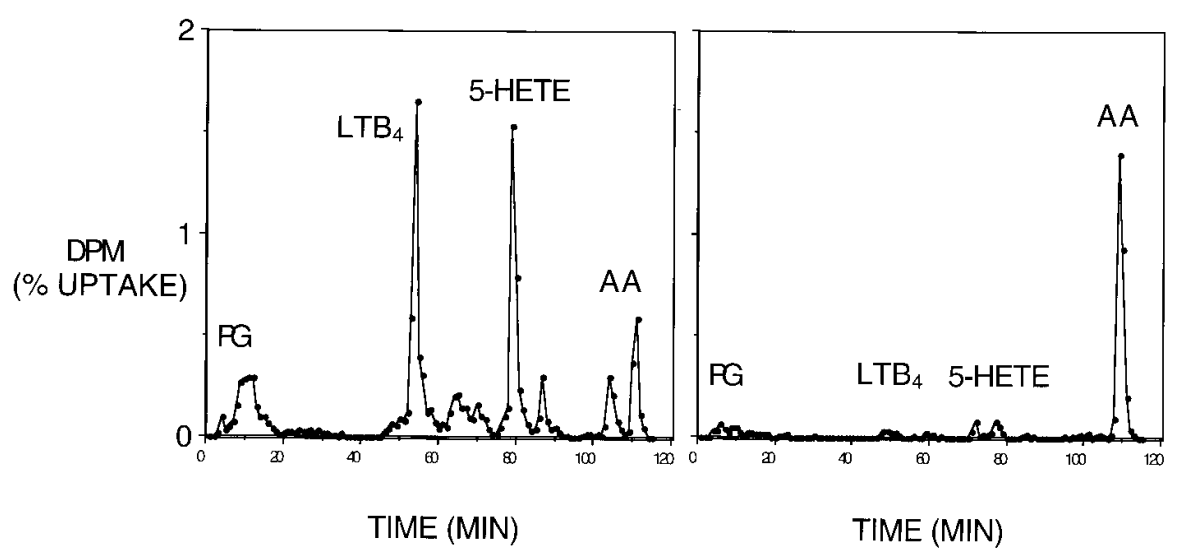

Figure 2. $\left[{ }^{3} \mathrm{H}\right]$ Eicosanoid profile of stimulated PMN from control and HIV-infected subjects. Prelabeled cells were incubated with $10 \mu \mathrm{M}$ A23187 for $30 \mathrm{~min}$. HPLC elution profiles are displayed for $\left[{ }^{3} \mathrm{H}\right] \mathrm{AA}$ metabolites synthesized by A23187-stimulated PMN from a representative normal subject and HIV-infected subject. Peaks were identified by coelution with authentic standards, and the products were expressed as a percentage of incorporated radioactivity. 
Table I. $\left[{ }^{3} H\right] A A$ Release in PMN from Control and $H I V$-infected Subjects

\begin{tabular}{llc}
\hline & \multicolumn{1}{c}{ Control } & HIV \\
\cline { 2 - 3 } & \multicolumn{1}{c}{$(\mathrm{dpm}$ [\% incorporated radioactivity] $)$} \\
\hline Experiment 1 & 1.35 & 3.27 \\
Experiment 2 & 0.62 & 4.55 \\
Experiment 3 & 3.3 & 1.44 \\
Mean +SEM & $1.9 \pm 0.99$ & $3.1 \pm 0.9$ \\
& & \\
\hline
\end{tabular}

PMN were incubated overnight, and then prelabeled for $1.5 \mathrm{~h}$ as described in Methods. Cells were stimulated with A23187 $(10 \mu \mathrm{M})$ for 30 min in the presence of $0.1 \%$ BSA. $\left[{ }^{3} \mathrm{H}\right] \mathrm{AA}$ was identified by co-elution with authentic standards, and expressed as a percentage of incorporated radioactivity. Control and HIV values were not statistically different.

in the AIDS population $(25,26)$. PMN from normal subjects demonstrated modest inhibition of $C$. neoformans growth in vitro. In contrast, PMN from HIV-infected subjects displayed reduced anticryptococcal activity manifested as increased colony numbers during the assay (Fig. 4).

$G$-CSF protocol patient population. The HIV group consisted of seven males and one female, with a mean age of $38.9 \pm 3.0 \mathrm{yr}$. The hematologic and viral characteristics of these eight HIV-infected subjects are shown in Table II. The mean CD4 count was $40.9 \pm 9.4 \mathrm{~cm}^{2}$. There was a marked increase in white cell count and absolute PMN count after G-CSF treatment. This peaked at day 4 , and was still elevated at approximately twice day 1 levels on day 7. As expected, the HIV load

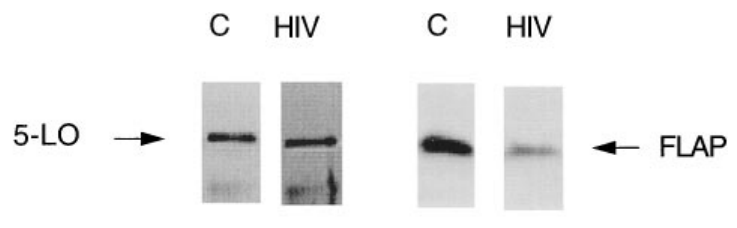

$$
\begin{aligned}
& \text { RELATIVE } \\
& \text { EXPRESSION } \\
& \text { (\% OF } \\
& \text { NORMAL PMN) }
\end{aligned}
$$

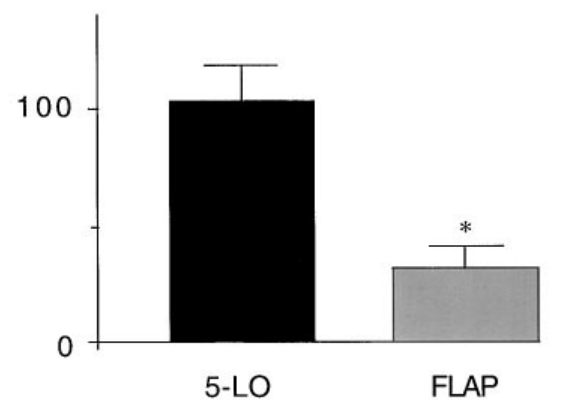

Figure 3. 5-LO and FLAP expression in PMN from control and HIVinfected subjects. Equal amounts ( $20 \mu \mathrm{g}$ of protein) of crude cellular lysate from PMN were subjected to immunoblot analysis for 5-LO and FLAP as described in Methods. (Top) Representative autoradiograph of a Western blot demonstrating the amount of 5-LO (left) and FLAP (right) in PMN from control and HIV-infected subjects. (Bottom) Relative expression of 5-LO and FLAP in PMN from controls and HIV-infected subjects, as assessed by densitometry and expressed as a percent of values derived from cells from normal subjects. Data represent the mean \pm SEM from $n=8$ subjects. ${ }^{*} P<$ 0.001 .

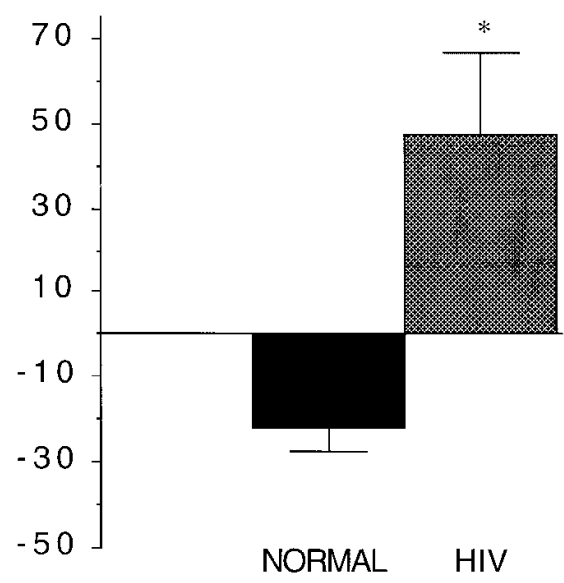

Figure 4. C. neoformans growth in the presence of PMN from controls and HIV-infected subjects. C. neoformans was incubated with PMN for $48 \mathrm{~h}$ as described in Methods. After cell lysis with $0.1 \%$ Triton-X, CFU were determined by serial dilution of cell supernatants and lysates on Sabouraud's dextrose agar. The data are presented as a percent change of $C$. neoformans growth compared with control medium (percent change CFU). Values given are the mean \pm SEM from $n=5$ subjects. $* P<0.05$.

was unchanged after G-CSF treatment (pretherapy 176.4 \pm 86 $10^{3}$, posttherapy $125.9 \pm 53.110^{3}$ particles bDNA). No significant side effects of G-CSF administration were reported.

Effect of G-CSF treatment on AA metabolism of PMN from $H I V$-infected subjects. We studied PMN before, during, and after $5 \mathrm{~d}$ of treatment with $300 \mu \mathrm{g}$ G-CSF administered subcutaneously. On the fourth day of treatment with G-CSF in vivo, there was a dramatic increase in LT synthesis detected in vitro (Fig. 5), as compared to the markedly reduced baseline levels. This increase in 5-LO metabolism had waned by day 7, and although there was a trend towards higher values than on day 1 , it was not statistically significant. To determine if this effect was selective for the 5-LO pathway, we determined COX metabolism in PMN from HIV-infected subjects in parallel with that of 5-LO metabolism. As compared with day 1 levels immunoreactive $\mathrm{PGE}_{2}$ synthesis was likewise increased following treatment of subjects with G-CSF on day $4(326.6 \pm 63.3 \%$ of

Table II. Laboratory Values for the HIV Patient Population

\begin{tabular}{lcccccccc}
\hline & \multicolumn{3}{c}{ WCC $\left(10^{3}\right)$} & & \multicolumn{3}{c}{ ANC $\left(10^{3}\right)$} \\
\cline { 2 - 4 } \cline { 7 - 8 } & No. & day 1 & day 4 & day 7 & day 1 & day 4 & day 7 \\
\hline 1 & 6.2 & 20.1 & 9.4 & & 4.5 & 16.7 & 7.6 \\
2 & 5.9 & 21.2 & 10 & & 2.7 & 16.7 & 6.8 \\
3 & 3.5 & 20 & 14 & & 1.6 & 17.8 & 10.6 \\
4 & 3.5 & 11.1 & 7.1 & & 1.75 & 6.9 & 5.0 \\
5 & 4.7 & 19.4 & 14.9 & & 1.7 & 15.5 & 8.9 \\
6 & 12.8 & 30.4 & 6.9 & & 11.6 & 29.2 & 5.2 \\
7 & 4.8 & 18.8 & 8.4 & & 2.9 & 16.4 & 5.7 \\
8 & 2.7 & 14.1 & 7 & & 1.6 & 11.4 & 4.5 \\
Mean \pm SEM & $5.5 \pm 1.1$ & $19.4 \pm 2.0$ & $9.7 \pm 1.1$ & $3.6 \pm 1.2$ & $16.3 \pm 2.2$ & $6.8 \pm 0.8$
\end{tabular}

ANC, absolute neutrophil count; WCC, white cell count. 


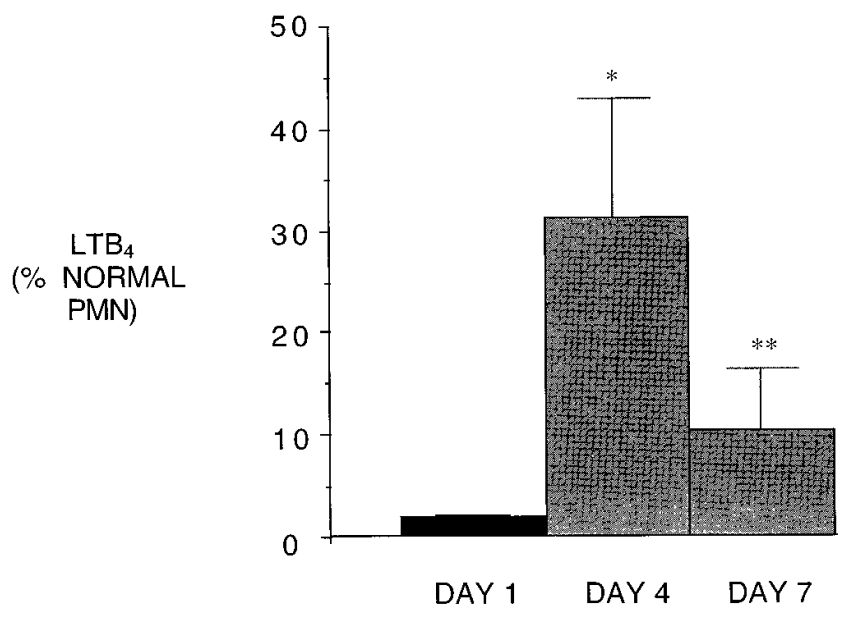

Figure 5. Effect of in vivo G-CSF therapy on PMN LT synthesis in HIV-infected subjects. PMN were isolated on days 1, 4, and 7 of the study protocol as described in Methods, and plated at $1 \times 10^{6} / \mathrm{ml}$ in Iscove's medium. Maximal $\mathrm{LTB}_{4}$ release was determined after stimulation with A23187 $(10 \mu \mathrm{M})$ for $30 \mathrm{~min}$ at $37^{\circ} \mathrm{C}$. Medium was analyzed for $\mathrm{LTB}_{4}$ by EIA and the value obtained for HIV-infected subjects was expressed as a percent of the value observed for control subjects $(n=8)(2,846.7 \pm 536.3) . * P=0.05$ vs. day $1 ; * * P=0.05$ vs. day 4 . $\mathrm{LTB}_{4}$ values were day $1,51.2 \pm 0 \mathrm{pg} / 10^{6}$ cells (these levels were undetected and taken as the lower limit of the assay sensitivity); day 4 , $886.0 \pm 329.4 \mathrm{pg} / 10^{6}$ cells; day $7,287.4 \pm 170.6 \mathrm{pg} / 10^{6}$ cells.

day 1$)$, and day $7(228.3 \pm 62.9 \%$ of day 1$)$. This suggested that an increase in AA release might account, at least in part, for the increases in both LT and PG product formation. This was indeed the case, since day $7 \mathrm{PMN}$ prelabeled with $\left[{ }^{3} \mathrm{H}\right] \mathrm{AA}$ demonstrated increased total release of radioactivity. Fig. 6 shows a HPLC profile which exhibits increased quantities of radiolabeled free AA, 5-LO products, and COX products synthesized by PMN after in vivo G-CSF treatment.

Effect of G-CSF treatment on 5-LO, FLAP, and cPLA $A_{2}$ expression in PMN from $H I V$-infected subjects. We next examined the effect of G-CSF treatment on the PMN expression of the two proteins, 5-LO and FLAP, essential for LT synthesis. Both FLAP and 5-LO expression, were increased on day 4 compared with day 1 (Fig. 7). This increase in protein expression was maintained on day 7 . In addition, we examined the expression of $\mathrm{cPLA}_{2}$ (data not shown). G-CSF also increased
cPLA $A_{2}$ expression (day 4: $210.7 \pm 31 \%$ of day 1 ; day $7: 214.4 \pm$ $43.7 \%$ of day $1, n=4, P=0.05)$ to the same extent as 5-LO. This increased $\mathrm{cPLA}_{2}$ expression correlated with the increase in AA release. These observations suggest that increased 5-LO and FLAP expression on day 4 and day 7 account for the augmented LT synthetic capacity. Increased $\mathrm{cPLA}_{2}$ expression and $\mathrm{AA}$ release may also contribute, in part, to the increase in 5-LO metabolites after G-CSF therapy. The decrease in LT synthesis on day 7 compared with day 4 may be explained by a decrease in $\mathrm{cPLA}_{2}$ activity (AA release), or activity of the 5-LO enzyme, since expression of 5-LO, FLAP, and $\mathrm{CPLA}_{2}$ proteins were unchanged.

Effect of G-CSF treatment on anticryptococcal activity of $P M N$ from $H I V$-infected subjects. To determine the functional significance of this increase in PMN 5-LO metabolism from HIV-infected subjects after G-CSF therapy, we determined their antifungal activity. PMN anticryptococcal activity in vitro was determined before (day 1) and after (day 7) treatment of HIV-infected subjects with G-CSF. As a result of G-CSF treatment, PMN shifted from a growth-promoting to a growth-suppressive milieu for C. neoformans (Fig. 8).

Role of increased LT synthesis in the G-CSF effect on PMN anticryptococcal activity. The above observations indicate that G-CSF treatment of HIV-infected subjects augments PMN 5-LO metabolism, and increases PMN anticryptococcal activity in parallel. The next logical step was to determine if the increase in anticryptococcal activity was dependent on increased PMN LT synthesis. We harvested PMN on day 7 from the HIV-infected subjects who were treated with G-CSF, and incubated the cells with a LT synthesis inhibitor, the FLAP antagonist MK-886. Notably, MK-886 substantially abrogated the enhanced anticryptococcal activity observed after G-CSF treatment (Fig. 9). Furthermore, when we treated PMN from control subjects with MK-886, we demonstrated reduced anticryptococcal activity (control PMN $-49.1 \pm 22.1 \%$ change in $C$. neoformans growth compared with control medium; MK-886 treated $7.8 \pm 0.37 \%, n=3, P=0.05)$. This indicates that the increased LT synthetic capacity of PMN from G-CSF-treated subjects was at least partially responsible for the augmented antifungal capacity.

\section{Discussion}

Recruitment of circulating PMN to tissues is an important second line of defense against microorganisms that augments the
DAY 1

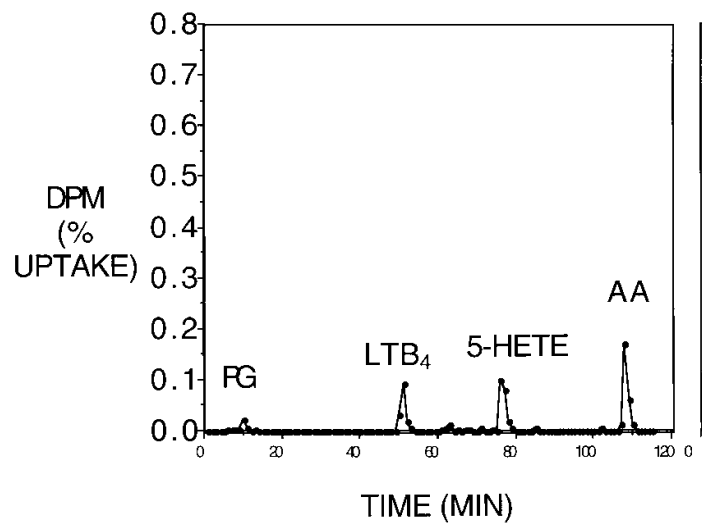

DAY 7

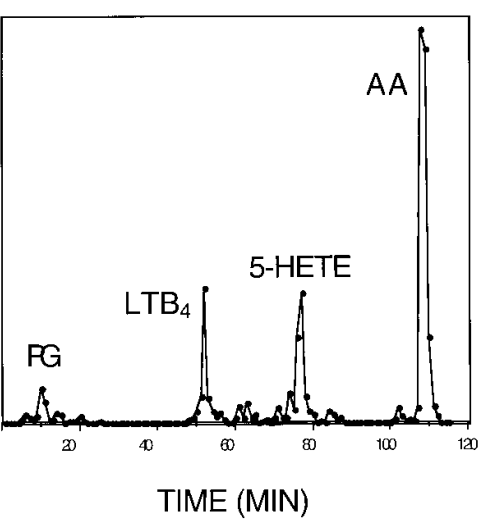

Figure 6. $\left[{ }^{3} \mathrm{H}\right]$ Eicosanoid profile of stimulated PMN from HIV-infected subjects before and after therapy with G-CSF. Prelabeled cells were incubated with $10 \mu \mathrm{M}$ A23187 for $30 \mathrm{~min}$, as described in the legend to Fig. 2. HPLC elution profiles are displayed for $\left[{ }^{3} \mathrm{H}\right]$ AA metabolites synthesized by A23187-stimulated PMN from a representative HIV-infected subject on day 1 and a HIV-infected subject on day 7. 


\section{A}

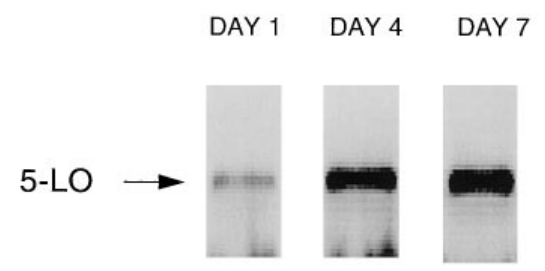

RELATIVE
EXPRESSION
(\% OF DAY 1)

B
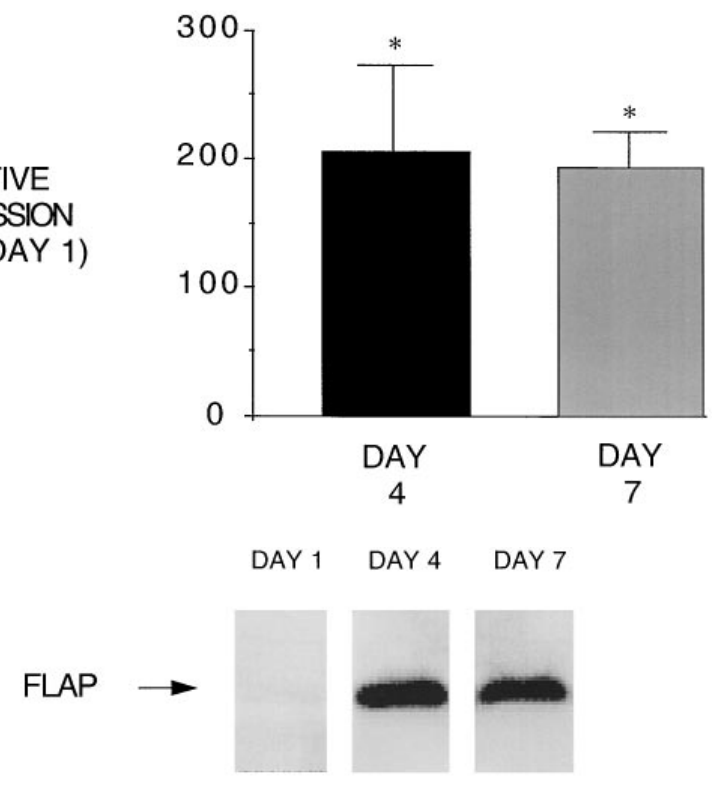

7
C. neoformans

$\mathrm{CFU}$
$\%$ CHANGE in

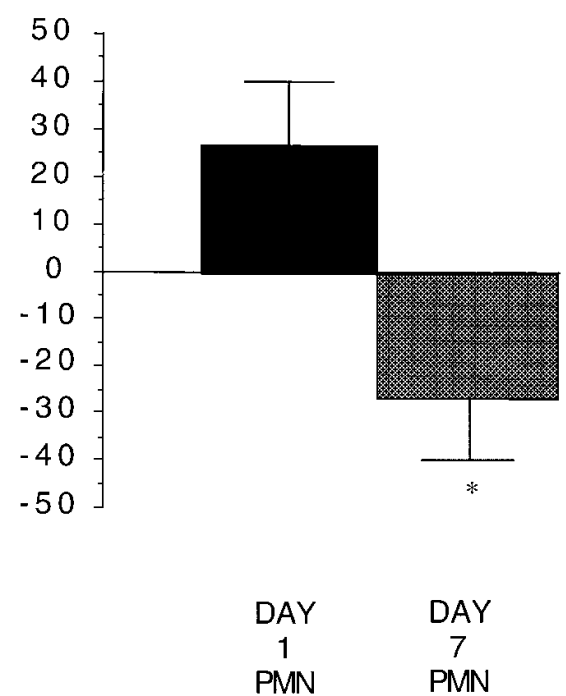

Figure 8. Effect of in vivo G-CSF therapy on PMN anticryptococcal activity in HIV-infected subjects. PMN were isolated on protocol days 1 and 7. C. neoformans was incubated with PMN for $48 \mathrm{~h}$ as described in Methods. After cell lysis with $0.1 \%$ Triton-X, CFU were determined by serial dilution of cell supernatants and lysates on Sabouraud's dextrose agar. The data are presented as a percent change of C. neoformans growth compared with control medium. Data represent the mean \pm SEM from $n=5$ subjects. $* P=0.02$.

termine if PMN exhibited the same defect. In fact, the defect was even more profound than that seen in AM.

This is the first report of reduced 5-LO metabolism in PMN from HIV-infected subjects. This defect is specific for the LT synthetic pathway and appears to be related to a decrease in FLAP expression. Importantly, this metabolic abnormality was associated with reduced antifungal activity of PMN in vitro. Treatment of subjects with G-CSF in vivo augmented

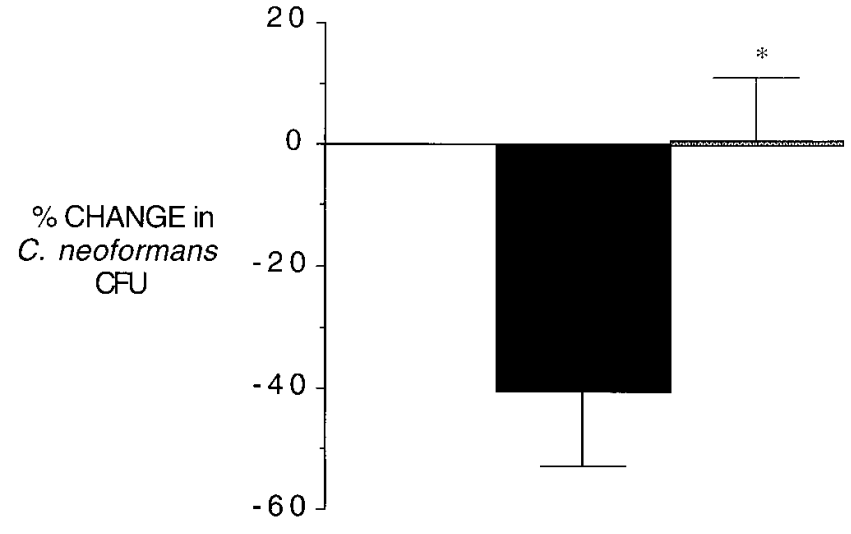

CONIROL MK-886

\section{DAY 7}

Figure 9. Role of 5-LO metabolism in the increased anticryptococcal activity of PMN from HIV-infected subjects after G-CSF therapy. PMN were harvested on day 7 of the protocol. C. neoformans was incubated with PMN for $48 \mathrm{~h}$, with or without the LT synthesis inhibitor MK-886. C. neoformans growth was determined as described above. Data represent the mean \pm SEM from $n=3$ subjects. $* P=0.05$. 
PMN 5-LO metabolism, with an associated increase in 5-LO and especially FLAP expression. The resulting increase in LT synthesis was associated with improved antifungal activity in vitro. These results provide insight into the mechanisms whereby G-CSF administration might augment PMN function. On the basis of recent work indicating that LT-deficient mice have increased susceptibility to pneumonia (13), we suggest that boosting PMN LT synthesis in subjects with HIV infection may ameliorate impaired antimicrobial defense in AIDS.

AM from HIV-infected subjects with low CD4 counts demonstrated reduced 5-LO and FLAP expression, whilst AM from HIV-infected subjects with normal CD4 counts demonstrated only reduced 5-LO expression (14). FLAP expression in AM correlated with the CD4 counts in these HIV-infected subjects. This led us to speculate that direct HIV infection of AM resulted in reduced 5-LO expression, while reduced numbers of CD4 cells resulted in reduced FLAP expression. This hypothesis is further supported by the evidence that AM from a CD4 $\mathrm{T}$ cell-depleted murine model demonstrate reduced 5-LO metabolism and FLAP expression (Coffey, M., S. Phare, M. Peters-Golden, and G. Huffnagle, manuscript submitted for publication) and that mediators released by activated blood lymphocytes upregulate 5-LO metabolism in peripheral blood monocytes (27). PMN, which are not susceptible to direct HIV infection, also demonstrate reduced FLAP expression with reduced LT synthetic capacity, consistent with the liklehood that reduced elaboration of mediators by CD4 T cells may be responsible for their reduced 5-LO metabolism as well.

A novel feature of this study was the attempt to upregulate defective PMN function by treating HIV-infected subjects with $\mathrm{G}-\mathrm{CSF}$. This growth factor is used in neutropenic patients to boost the PMN count (3). Additionally, there is evidence that levels of G-CSF, which is released by activated macrophages among other cells, are reduced in HIV-infected subjects (28). In vivo treatment of HIV-infected subjects with G-CSF increased PMN LT synthetic capacity. This was accompanied by an increase in PMN FLAP and 5-LO expression. Throughout this report, LT synthetic capacity, protein expression, and antifungal activity were expressed per million PMN. Since G-CSF also substantially increased PMN cell count, all of these cellular effects would be expected to be magnified in vivo. GM-CSF has been shown to increase PMN 5-LO metabolism in vitro $(16,17,29)$ and augment urinary $\mathrm{LTE}_{4}$ levels after in vivo therapy $(30,31)$. However, the effect of G-CSF on 5-LO metabolism is less well studied. Our study is the first report of an effect of in vivo treatment with a CSF on cellular 5-LO metabolism.

Another important aspect of this study is our investigation of the functional significance of this defect in PMN 5-LO metabolism. C. neoformans is a common opportunistic organism causing disseminated disease in AIDS, and normal PMN have in vitro anticryptococcal activity (32). Although neutropenia is not a classic presentation in AIDS, it may be occurring more frequently with increased use of aggressive antiretroviral regimes with their attendant bone marrow suppression. Furthermore, with the combination of lymphocyte and macrophage dysfunction in AIDS, the PMN may become more relevant in preventing fungal dissemination from the lung. We demonstrate defective killing of $C$. neoformans by PMN from HIV-infected subjects compared with control PMN. G-CSF treatment of $\mathrm{HIV}$-infected subjects boosted PMN antifungal activity in vitro in parallel with increases in 5-LO metabolism and 5-LO and FLAP expression. Furthermore, inhibiting LT synthesis with the FLAP inhibitor, MK-886, partially reversed the increased antifungal activity of PMN from HIV-infected subjects attributed to G-CSF treatment. It should be noted that we did not study anticryptococcal activity, during G-CSF therapy on day 4, when 5-LO metabolism was maximal. Therefore, it is likely that we have underestimated the effect of G-CSF therapy, and hence LT, on PMN anticryptococcal activity. These observations provide important insight into one mechanism whereby $\mathrm{G}-\mathrm{CSF}$ augments PMN function when administered in vivo. Indeed, the effects of G-CSF on neutrophil function, such as enhanced phagocytosis, microbiocidal activity, and delayed apoptosis, are also the effects of LT on PMN function $(11,13,33)$.

These data have important clinical implications for the management of HIV-infected subjects with opportunistic infections. In addition to the role of LT in the killing of microbes, LT increase complement killing of HIV (34). In addition, $\mathrm{LTB}_{4}$ and 5-HETE have been shown to increase natural killer cell activity, which helps eliminate virus-infected cells (35). 5-LO products, including $\mathrm{LTB}_{4}$ and 5-HETE, are well known to recruit PMN to sites of infection and to activate their antimicrobial function against both fungi and bacteria. The predisposition to pulmonary infections with reduced LT synthetic capacity has a precedent not only in 5-LO knockout mice, but in other animal models, including protein-calorie malnutrition (36), vitamin $\mathrm{D}_{3}$ deficiency (37), and infancy (38), as well as in human diabetes mellitus (39). Treatment of HIVinfected subjects with G-CSF during opportunistic infections may enhance clearance of opportunistic and nonopportunistic organisms. Furthermore, preliminary data suggest that the addition of G-CSF to standard antibiotic therapy may enhance the treatment of community acquired pneumonia in non-HIV patients (40).

In summary, this defect in PMN from HIV-infected subjects compounds a similar defect in AM LT synthesis (14) and the better-recognized reduction in CD4 T cell number and function. Reduced mediator release from CD4 cells may result in defective killing of microorganisms by phagocytic cells. Replacement of growth factors such as G-CSF has the potential to augment defective PMN LT synthesis and antimicrobial activity.

\section{Acknowledgments}

This work was supported by a grant from the Amgen Pharmaceutical Company, the American Lung Association of Michigan and the General Clinical Research Center at the Univ. of Michigan (M01RR00042). M.J. Coffey was the recipient of National Institutes of Health Clinical Investigator Development Award (HL-02810). M. Peters-Golden was supported by the National Institutes of Health (R01-HL471) and a Career Investigator Award from the American Lung Association.

\section{References}

1. Centers for Disease Control. 1986. Classification system for human T-lymphotropic virus type III/lymphadenopathy-associated virus infections. Ann. Intern. Med. 105:234-237.

2. Roilides, E., S. Mertins, J. Eddy, T.J. Walsh, P.A. Pizzo, and M. Rubin. 1990. Impairment of neutrophil chemotactic and bactericidal function in children infected with human immunodeficiency virus type 1 and partial reversal after in vitro exposure to granulocyte-macrophage colony-stimulating factor. $J$. Pediatr. 117:531-540.

3. Roilides, E., T. Walsh, P. Pizzo, and M. Rubin. 1991. Granulocyte colony stimulation factor enhances the phagocytic and bactericidal activity of normal 
and defective human neutrophils. J. Infect. Dis. 163:579-583.

4. Pitrak, D., P. Bak, P. De Marais, R. Novak, and B. Andersen. 1993. Depressed neutrophil superoxide production in human immunodeficiency virus infection. J. Infect. Dis. 167:1406-1410.

5. Chen, T., R. Roberts, W. Kg, B. Ank, and E. Stiehm. 1993. Decreased superoxide anion and hydrogen peroxide production by neutrophils and monocytes in HIV-infected children and adults. Pediatr. Res. 34:544-550.

6. Hirschtick, R., J. Glassroth, M. Jordan, T. Wilcosky, J. Wallace, P. Kvale, N. Markowitz, M. Rosen, B. Mangura, P. Hopewell, et al. 1995. Bacterial pneumonia in persons infected with the human immunodeficiency virus. N. Engl. J. Med. 333:845-851.

7. Needleman, P., J. Turk, B.A. Jakschik, A.R. Morrison, and J.B. Lefkowith. 1986. Arachidonic acid metabolism. Annu. Rev. Biochem. 55:69-102.

8. Samuelsson, B., and C.D. Funk. 1989. Enzymes involved in the biosynthesis of leukotriene $B_{4}$. J. Biol. Chem. 264:19469-19472.

9. Henderson, W., Jr. 1987. Eicosanoids and lung inflammation. Am. Rev. Respir. Dis. 135:1176-1185.

10. Serhan, C., U. Lundberg, G. Weissmann, and B. Samuelsson. 1984. Formation of leukotrienes and hydroxy acids from human neutrophils and platelets exposed to monosodium urate. Prostaglandins. 27:563-581.

11. Demitsu, T., H. Katayama, T. Saito-Taki, H. Yaoita, and M. Nakano. 1989. Phagocytosis and bactericidal action of mouse peritoneal macrophages treated with leukotriene $\mathrm{B}_{4}$. Int. J. Immunopharmacol. 11:801-808.

12. Marder, P., J. Sawyer, L. Froelich, L. Mann, and S. Spaethe. 1995. Blockade of human neutrophil activation by 2-[2-propyl-3-[3-[2-ethyl-4-(4-fluorophenyl)-5-hydroxyphenoxy]propoxy]phenoxy]benzoic acid (LY29311), a novel leukotriene $\mathrm{B}_{4}$ receptor antagonist. Biochem. Pharmacol. 49:1683-1690.

13. Bailie, M., J. Standiford, L. Laichalk, M. Coffey, R. Strieter, and M. Peters-Golden. 1996. Leukotriene-deficient mice manifest enhanced lethality from klebsiella pneumonia in association with decreased alveolar macrophage phagocytic and bactericidal activities. J. Immunol. 157:5221-5224.

14. Coffey, M., S. Phare, P. Kazanjian, and M. Peters-Golden. 1996. Reduced 5-lipoxygenase metabolism in alveolar macrophages from subjects infected with the human immunodeficiency virus. J. Immunology. 157:393-399.

15. Lieschke, G., and A. Burgess. 1992. Granulocyte colony-stimulating factor and granulocyte-macrophage colony-stimulating factor. N. Eng. J. Med. 327: 28 .

16. Pouliot, M., P. McDonald, L. Khamzina, P. Borgeat, and S. McColl. 1994. Granulocyte-macrophage colony-stimulating factor enhances 5-lipoxygenase levels in human polymorphonuclear leukocytes. J. Immunol. 152:851-858.

17. Pouliot, M., P. McDonald, P. Borgeat, and S. McColl. 1994. Granulocyte/macrophage colony-stimulating factor stimulates the expression of the 5-lipoxygenase-activating protein (FLAP) in human neutrophils. J. Exp. Med. 179:1225-1232.

18. Sullivan, R., J. Griffin, E. Simons, A. Schafner, T. Meshulam, J. Fredette, A. Maas, A.-S. Gadenne, J. Leavitt, and D. Melnick. 1987. Effects of recombinant human granulocyte and macrophage colony-stimulating factors on signal transduction pathways in human granulocytes. J. Immunol. 139:34223430.

19. Mellors, J., L. Kingsley, C. Rinaldo, J. Todd, B. Hoo, R. Kokka, and P. Gupta. 1995. Quantitation of HIV-1 RNA in plasma predicts outcome after seroconversion. Ann. Intern. Med. 122.573-579.

20. Peters-Golden, M., R.W. McNish, R. Hyzy, C. Shelly, and G.B. Toews. 1990. Alterations in the pattern of arachidonate metabolism accompany rat macrophage differentiation in the lung. J. Immunol. 144:263-270.

21. Coffey, M., M. Peters-Golden, J. Fantone, and P. Sporn. 1992. Membrane association of active 5-lipoxygenase in resting cells: evidence for novel regulation of the enzyme in the rat alveolar macrophage. J. Biol. Chem. 267: $570-576$

22. Laemmli, U. 1970. Cleavage of structural proteins during the assembly of the head of bacteriophage T4. Nature. 227:680-685.

23. Peters-Golden, M., and R. McNish. 1993. Redistribution of 5-lipoxygenase and cytosolic phospholipase $\mathrm{A}_{2}$ to the nuclear fraction upon macrophage activation. Biochem. Biophys. Res. Commun. 196:18-24.

24. Chen, G., J. Curtis, C. Mody, P. Christensen, L. Armstrong, and G. Toews. 1994. Effect of granulocyte-macrophage colony-stimulating factor on rat alveolar macrophage anticryptococcal activity in vitro. J. Immunol. 152:724-834

25. Chuck, S., and M. Sande. 1989. Infections with Cryptococcus neoformans in the acquired immunodeficiency syndrome. N. Eng. J. Med. 321:794-799.

26. Levitz, S. 1991. The ecology of cryptococcus neoformans and the epidemiology of cryptococcosis. Rev. Infect. Dis. 13:1163-1169.

27. Ring, W., C. Riddick, J. Baker, D. Munafo, and T. Bigby. 1996. Lymphocytes stimulate expression of 5-lipoxygenase and its activating protein in monocytes in vitro via granulocyte-macrophage colony-stimulating factor and interleukin 3. J. Clin. Invest. 97:1293-1301.

28. Watari, K., S. Asamo, N. Shirafuji, H. Kodo, K. Ozawa, F. Takakuet, and S. Kamachi. 1989. Serum granulocyte colony-stimulating factor levels in healthy volunteers and patients with various disorders as estimated by enzyme immunoassay. Blood. 73:117-122.

29. McColl, S., E. Krump, P. Naccache, P. Poubelle, M. Braquet, and P. Borgeat. 1991. Granulocyte-macrophage colony-stimulating factor increases the synthesis of Leukotriene $\mathrm{B}_{4}$ by human neutrophils in response to plateletactivating factor. J. Immunol. 146:1204-1211.

30. Denzlinger, C., A. Kapp, M. Grimberg, H. Gerhartz, and W. Wilmanns. 1990. Enhanced endogenous leukotriene biosynthesis in patients treated with granulocyte-macrophage colony-stimulating factor. Blood. 76:1765-1770.

31. Denzlinger, C., W. Tetzloff, H. Gerhartz, R. Pokorny, S. Sagebiel, C. Haberl, and W. Wilmanns. 1993. Differential activation of the endogenous leukotriene biosynthesis by two different preparations of granulocyte-macrophage colony-stimulating factor in healthy volunteers. Blood. 81:2007-2013.

32. Chaturvedi, V., B. Wong, and S. Newman. 1996. Oxidative killing of cryptococcus neoformans by human neutrophils. J. Immunol. 156:3836-3840.

33. Herbert, M.-J., T. Takano, H. Holthofer, and H. Brady. 1996. Sequential morphologic events during apoptosis of human neutrophils: modulation by lipoxygenase-derived eicosanoids. J. Immunol. 157:3105-3115.

34. Miyamoto, K., G. McKinley, M. Lange, C. Stavropoulos, H. Matsumoto, S. Moriya, and Y. Inada. 1992. Effects of sho-saiko-to (SST) on production of prostaglandin $\mathrm{E}_{2}\left(\mathrm{PGE}_{2}\right)$, leukotriene $\mathrm{B}_{4}\left(\mathrm{LTB}_{4}\right)$ and superoxide from peripheral monocytes and neutrophils isolated from HIV infected individuals. Int Conf. AIDS. 8:A60. (Abstr.)

35. Chang, K., H. Saito, I. Tatsuno, Y. Tamura, and S. Yoshida. 1991. Role of 5-lipoxygenase products of arachidonic acid in cell-to-cell interaction between macrophages and natural killer cells in rat spleen. J. Leukocyte Biol. 50: 273-278.

36. Skerrett, S., W. Henderson, and T. Martin. 1990. Alveolar macrophage function in rats with severe protein calorie malnutrition: arachidonic acid metabolism, cytokine release, and antimicrobial activity. J. Immunol. 144:10521061

37. Bar-Shavit, Z., D. Noff, S. Edelstein, M. Meyer, S. Shibolet, and R. Goldman. 1981. 1,25-dihydroxyvitamin D3 and the regulation of macrophage function. Calcif. Tissue Int. 33:673-676.

38. Lu, M-C., D. Hostetler, N. Robinson, F. Derksen, and M. PetersGolden. 1995. Age-related increases in 5-lipoxygenase activity of cattle alveolar macrophages. Am. J. Physiol. 271:L547-L554.

39. Greco, N., M. Milks, and R. Panganamala. 1990. Metabolism of arachidonic acid in neutrophils from alloxan-diabetic rabbits. Prostaglandins Leukotrienes Essential Fatty Acids. 42:201-208.

40. Nelson, S., S. Farkas, N. Fotheringham, H. Ho, T. Marrie, and H. Movahhed. 1996. Filgrastim in the treatment of hospitalized patients with community acquired pneumonia. Am. J. Respir. Crit. Care. Med. 153S:A535. (Abstr.) 